\title{
PD-LI Expression in Endometrial Serous Carcinoma and Its Prognostic Significance
}

\author{
Tao Zhang $\mathbb{D}^{1, *}$ \\ Qin $\mathrm{Liu}^{2} *$ \\ Yingfan Zhu' \\ Songfa Zhang ${ }^{3}$ \\ Qiaohua Peng ${ }^{3}$ \\ Amanda Louise Strickland ${ }^{4}$ \\ Wenxin Zheng $\mathbb{D}^{4,5}$ \\ Feng Zhou ${ }^{2}$ \\ 'Department of Gynecology, Women's \\ Hospital, School of Medicine, Zhejiang \\ University, Hangzhou, Zhejiang, People's \\ Republic of China; ${ }^{2}$ Department of \\ Pathology, Women's Hospital, School of \\ Medicine, Zhejiang University, Hangzhou, \\ Zhejiang, People's Republic of China; \\ ${ }^{3}$ Department of Oncology, Women's \\ Hospital, School of Medicine, Zhejiang \\ University, Hangzhou, Zhejiang, People's \\ Republic of China; ${ }^{4}$ Department of \\ Pathology, Northwestern University, \\ Feinberg School of Medicine, Chicago, IL, \\ USA; ${ }^{5}$ Department of Pathology, \\ University of Texas Southwestern \\ Medical Center, Dallas, TX, USA \\ *These authors contributed equally to \\ this work
}

Purpose: Programmed death-ligand 1 (PD-L1) has been widely used as a prognostic biomarker and an immunotherapeutic target in numerous cancers, but information on the clinical significance of its expression in endometrial serous carcinoma (ESC) is largely lacking. Here, we evaluate the predictive value of PD-L1 expression in ESC.

Materials and Methods: A total of 79 cases of ESC accessioned between January 2003 and September 2015 were selected for further analysis. PD-L1 expression was evaluated in whole tissue sections of these cases by using the tumor proportion score (TPS, cut-off $1 \%$ ) and combined positive score (CPS, cut-off 1) scoring methods.

Results: Overall, there was a heterogeneous expression of PD-L1, focal or patchy, in ESCs. PD-L1 positivity was observed in $43.0 \%$ of ESCs by TPS and $73.4 \%$ of ESCs by CPS. Kaplan-Meier survival analysis showed that patients with PD-L1-positive tumors suffered significantly worse OS and PFS, when compared with PD-L1 negative tumors (log-rank $p=$ 0.037 and $p=0.003$, respectively). In contrast, PD-L1 positivity by CPS within the ESC cases showed no statistical significance for OS and PFS (log-rank $p=0.720$ and $p=0.928$, respectively). Multivariate Cox analysis showed that PD-L1 positivity by TPS was significantly associated with PFS $(\mathrm{HR}=1.921, \mathrm{p}=0.039)$ but not $\mathrm{OS}(\mathrm{HR}=1.229, \mathrm{p}=0.631)$.

Conclusion: PD-L1 expression is frequently found in ESC, suggesting a potential role of the PD-1/PD-L1 pathway as a potential therapeutic target for these tumors. PD-L1 expression by TPS also serves as a negative prognostic marker in ESC and implies an unfavorable outcome.

Keywords: PD-L1, endometrial serous carcinoma, endometrial cancer, immune therapy

\section{Background}

Endometrial carcinoma is the 6th leading cause of cancer-related death among women worldwide. ${ }^{1}$ It can broadly be divided into two types: endometrioid carcinoma (type 1) and non-endometrioid carcinoma (type 2). Unlike type 1 carcinomas, type 2 carcinomas are highly aggressive and typically carry a poor prognosis. ${ }^{2}$ Endometrial serous carcinoma (ESC) is a type 2 endometrial cancer with a high mortality and recurrence rate. ${ }^{3}$ According to the Classification of Female Genital Tumors by World Health Organization (WHO 2020), ${ }^{4}$ ESCs are subclassified into pure and mixed groups. At present, patients with ESC are treated with radical hysterectomy and pelvic lymphadenectomy, omentectomy with or without adjuvant chemoradiation therapies. ${ }^{5}$ Regardless of therapy, the outcome of ESC patients tends to be poorer than patients with endometrioid carcinomas. ${ }^{6}$ Notably, it has been proposed in recent years that similar genetic landscape cancers even without the same tissue or origin can be classified together. ${ }^{7,8}$ According to this
Correspondence: Feng Zhou Department of Pathology, Women's Hospital, School of Medicine, Zhejiang University, Hangzhou City, Zhejiang Province, 310006, People's Republic of China

Tel +86-57I-89991702

Fax +86-57I-87061878

Email fungchew@zju.edu.cn 
classification, ESC and serous ovarian carcinoma can be considered as one category for similar cancer genetic drivers and progression mechanisms, ${ }^{9}$ such as TP53 mutation. ${ }^{2}$ Therefore, the treatment strategies of ovarian serous carcinoma carry significant potential value for ESC. Among different therapeutic strategies, novel immunotherapy such as Pembrolizumab gave the active response to advanced recurrent patients of ovarian serous carcinoma. ${ }^{10}$ As such, novel immunotherapeutic strategies need to be developed and established for patients with ESC. ${ }^{6}$

Programmed cell death receptor 1 (PD-1) and its ligand (PD-L1) are playing a progressively important role in our understanding of tumor immunology and in cancer treatment. Binding of PD-L1 to its receptor PD-1 leads to an inactivation of $T$ cells in a variety of cancers; thus, anti-PD -1/PD-L1 antibodies deregulate the adverse impact of tumor-infiltrating $\mathrm{T}$ cells, which in turn may reverse the tumor immune resistance. ${ }^{11,12}$ Several studies have investigated PD-L1 expression and its prognostic values in endometrial cancer, but most of such studies focused on endometrioid carcinomas without considering ESC as a separate or independent group. ${ }^{13-19}$ Of note, clinical trials with anti-PD-1 antibody (Pembrolizumab) have been performed in advanced recurrent endometrial cancer. Unfortunately, these trials did not separate ESCs from other histologic types of endometrial cancer. ${ }^{20}$

The relatively poorer outcome for patients with ESC using traditional treatment methods portends great importance in finding effective targeted immune. The main aim of our study is to investigate PD-L1 expression in and its prognostic value in ESC.

\section{Materials and Methods}

\section{Patients}

Female patients with a final histologic diagnosis of ESC between January 2003 and September 2015 in our Hospital were selected for further analysis. Hematoxylin and eosin (H\&E) and immunohistochemistry (IHC) stained slides were reviewed by at least 2 gynecologic pathologists. All tumors were classified according to the WHO (2020) Classification of Female Genital Tumors. ${ }^{4}$ Patients were clinically staged by using the FIGO (2018) system. Excluded cases included patients who had undergone their operations outside of our hospital or those with uterine tumor metastasis from other sources. Ultimately, 79 patients were included in the final cohort. Archived paraffin tissue blocks were obtained for all included cases. All related clinical and pathologic data including age, body mass index (BMI), serum CA125 level, stage, ascites or peritoneal wash status, myometrial invasion, lymph node metastasis, lymphovascular space invasion (LVSI), residual disease status, treatment, and clinical prognosis were retrieved from the database of clinical information system. Overall survival (OS) was defined as the time (months) between the date of surgery/diagnosis and the date of the death. Progression-free survival (PFS) was defined as the time (months) between the date of initial treatment and the date of progression to new tumor event. The normal upper limitation of serum CA125 was defined as $35 \mathrm{U} / \mathrm{mL}$. Optimal cytoreductive surgery of cancer staged III-IV was defined as $\leq 1 \mathrm{~cm}$ maximal diameter of the largest residual tumor nodule at initial treatment. ${ }^{3}$ Both the result of p16 and p53 were recorded. The study was approved by the Institutional Research Ethics Board at Zhejiang University School of Medicine Women's Hospital, China.

\section{Immunohistochemistry (IHC)}

In brief, $4 \mu \mathrm{m}$ thick formalin-fixed, paraffin-embedded whole tissue sections were dried fixed on positively charged slides for 1 hour at 56 to $60^{\circ} \mathrm{C}$. With antigen repaired, the slides were placed in the Autostainer Link 48 platform (Dako), where they were incubated with the monoclonal mouse anti-human PD-L1 antibody, clone 22C3 pharmDx (Dako, Santa Clara, CA), then the antimouse linker antibody and finally a substrate-chromogen solution (DAB). The incubation time and rinsing of slides between reagents was monitored. The reagent times were set in the Dako Link software. Slides were subsequently counterstained for 5 minutes with hematoxylin. Mounting was performed using non-aqueous, permanent mounting media. Positive and negative control tissue for the quality assurance of IHC was monitored.

\section{Analysis of PD-LI Expression}

Two pathologists independently reviewed and evaluated all the IHC-stained PD-L1 slides. Assessment of PD-L1 expression involved calculating the tumor proportion score (TPS) and combined positive score (CPS) as described previously. $^{21,22}$ Intra-tumoral and peri-tumoral immune cells, mainly lymphocytes and macrophages were counted. Of note, these cells were within one $20 \times$ field of the tumor nest or edge. PD-L1 positivity was defined by using a cutoff score $\geq 1 \%$ for TPS and $\geq 1$ for CPS. Of note, only the ESC areas were assessed in the cases of mixed ESC. 


\section{Statistical Analysis}

For the description of clinicopathological parameters, continuous variables are expressed as the mean \pm standard deviation (normally distributed) or median and 0-100th range (abnormally distributed), and categorical values are reported as frequencies. Chi-square or Fisher's exact test was used for cross-tables. Cox regression was used for multivariate analysis, with enter and forward selection. Kaplan-Meier survival analysis and Log rank test were used to assess the relationship between survival outcomes (OS and PFS) and PD-L1 expression. A significance level of 0.05 was used. IBM SPSS 20 (Armonk, NY) and GraphPad Prism 8 (San Diego, CA) were used for survival analysis.

\section{Results}

\section{Clinicopathological Characteristics of Patients}

Tissue samples of this study included 79 total hysterectomies and staging specimens. Among them, 45 cases were pure ESC and 34 were ESC mixed with endometrioid carcinoma. All pure ESCs and ESC areas in mixed ESCs were p16 block positive and demonstrated p53 mutant expression. Patient age ranged from 49 to 84 with the mean of $63 \pm 6.8$ years. Among the cases, 37 (46.8\%) of 79 were aged older than 63 years, 31 (39.2\%) had overweight (BMI $\left.\geq 25 \mathrm{~kg} / \mathrm{m}^{2}\right), 19(24.1 \%)$ had abnormal serum levels of CA125 (>35 U/mL).

Regarding clinical staging information, 38 (48.1\%) of the 79 cases had stage I disease, while $41(51.9 \%)$ had stage II or above. As for the other prognosis-related parameters, 29 (35.4\%) of 79 had deep myometrial invasion, 19 (24.1\%) showed evidence of LVSI, 7 (8.9\%) had more than $1 \mathrm{~cm}$ residual disease, and $10(12.7 \%)$ had positive malignant cells in ascites/peritoneal washes. Among all 79 cases, lymph node dissections were obtained in 70 cases, of which 16/70 (22.9\%) had lymph nodal metastasis. The information on this baseline data is summarized in Table 1.

\section{Correlation of PD-LI Expression with Clinicopathological Features in ESCs}

As mentioned, we examined the PD-L1 expression both in tumor cells and in tumor-infiltrating immune cells, including lymphocytes and macrophages. Overall, there was

Table I PD-LI Expression in Relation to Clinicopathological Parameters

\begin{tabular}{|c|c|c|c|c|c|c|c|}
\hline \multirow[t]{2}{*}{ Variable } & \multirow[t]{2}{*}{ Category } & \multicolumn{2}{|c|}{ TPS (n) } & \multirow[t]{2}{*}{ p-value } & \multicolumn{2}{|c|}{ CPS (n) } & \multirow[t]{2}{*}{ p-value } \\
\hline & & $<1 \%$ & $\geq \mathbf{I} \%$ & & $<1$ & $\geq \mathbf{I}$ & \\
\hline \multirow[t]{2}{*}{ Age (years) } & $\leq 63$ & 19 & 23 & $0.025^{\dagger}$ & 9 & 33 & 0.252 \\
\hline & $>63$ & 26 & II & & 12 & 25 & \\
\hline \multirow[t]{2}{*}{ BMI $\left(\mathrm{kg} / \mathrm{m}^{2}\right)$} & $<25$ & 26 & 22 & 0.532 & 11 & 37 & 0.359 \\
\hline & $\geq 25$ & 19 & 12 & & 10 & 21 & \\
\hline \multirow[t]{2}{*}{ CAI25 (U/mL) } & $\leq 35$ & 35 & 25 & 0.662 & 15 & 45 & 0.572 \\
\hline & $>35$ & 10 & 9 & & 6 & 13 & \\
\hline \multirow[t]{2}{*}{ Histotype } & Pure & 26 & 19 & 0.866 & 14 & 31 & 0.295 \\
\hline & Mixed & 19 & 15 & & 7 & 27 & \\
\hline \multirow[t]{2}{*}{ Stage } & I & 27 & II & $0.015^{\dagger}$ & 12 & 26 & 0.333 \\
\hline & II-IV & 18 & 23 & & 9 & 32 & \\
\hline \multirow[t]{2}{*}{ MI } & $<50 \%$ & 36 & 15 & $0.001^{\dagger}$ & 18 & 33 & $0.018^{\dagger}$ \\
\hline & $\geq 50 \%$ & 9 & 19 & & 3 & 25 & \\
\hline \multirow[t]{2}{*}{ LNM } & Negative & 40 & 23 & 0.060 & 17 & 37 & 0.529 \\
\hline & Positive & 6 & 10 & & 3 & 13 & \\
\hline \multirow[t]{2}{*}{ LVSI } & Negative & 40 & 20 & $0.002^{\dagger}$ & 18 & 42 & 0.222 \\
\hline & Positive & 5 & 14 & & 3 & 16 & \\
\hline \multirow[t]{2}{*}{ Surgery } & $\mathrm{RD} \leq \mathrm{Icm}$ & 43 & 29 & 0.133 & 19 & 53 & 1.000 \\
\hline & $\mathrm{RD}>\mathrm{Icm}$ & 2 & 5 & & 2 & 5 & \\
\hline \multirow[t]{2}{*}{ Ascites } & Negative & 42 & 27 & 0.090 & 19 & 50 & 1.000 \\
\hline & Positive & 3 & 7 & & 2 & 8 & \\
\hline
\end{tabular}

Note: ${ }^{\dagger} p<0.05$, Chi-square or Fisher's exact test was used.

Abbreviations: PD-LI, Programmed death-ligand I; TPS, tumor proportion score; CPS, combined positive score; MI, Myometrial invasion; LNM, Lymph node metastasis; LVSI, Lymphovascular space invasion; RD, residual disease. 


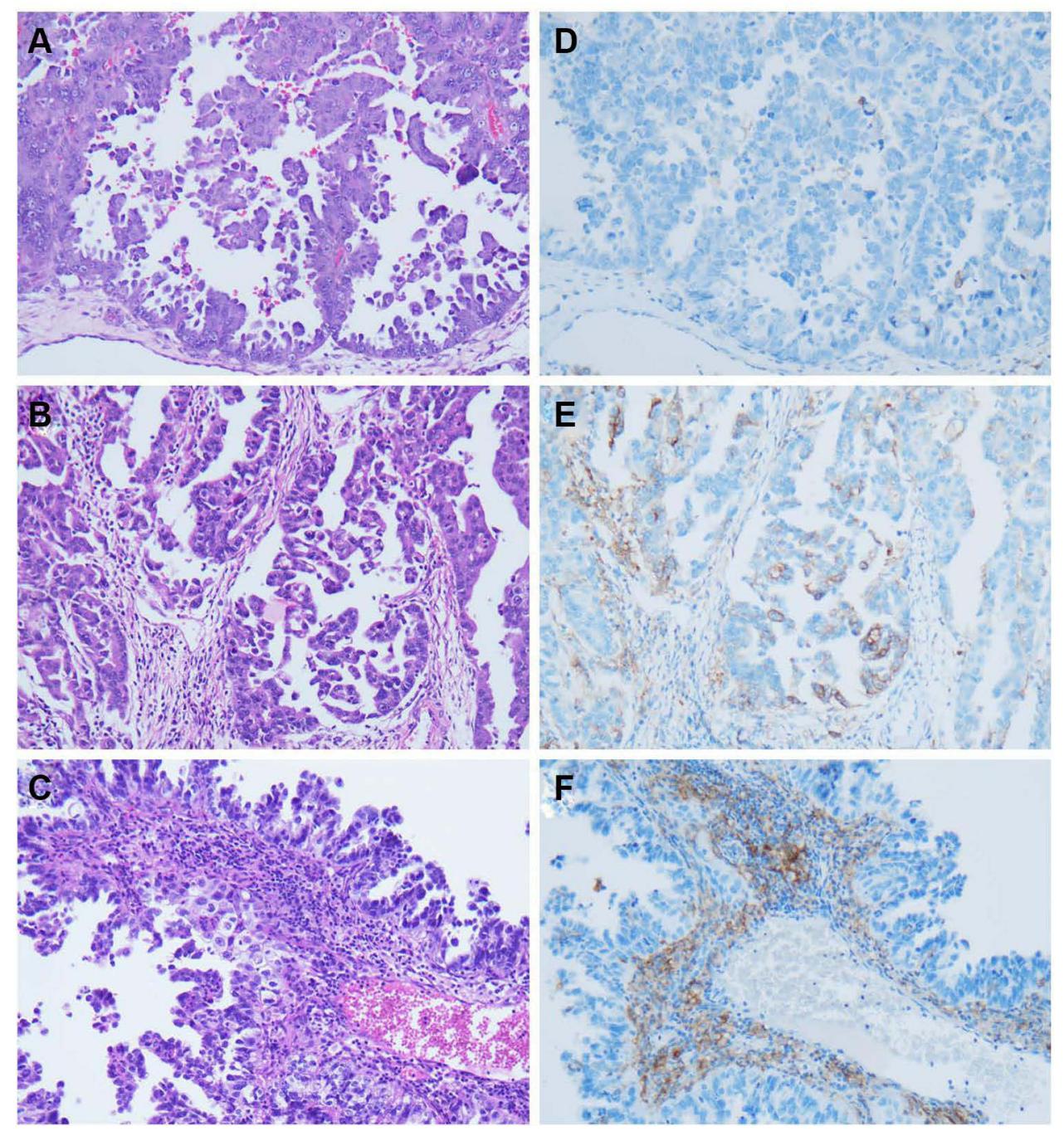

Figure I PD-LI expression in endometrial serous carcinoma. (A-C) Representative area of endometrial serous carcinoma at medium power (H\&E, 200x); (D) Corresponding negative PD-LI expression;(E) Corresponding PD-LI expression in tumor cells; (F) Corresponding PD-LI expression in immune cells.

a heterogeneous expression of PD-L1, focal or patchy, in ESCs (Figure 1). Using TPS, PD-L1 expression was seen in $43.0 \%(n=34)$ of the 79 ESC cases studied. Using CPS, PD-L1 expression was seen in $73.4 \%(n=58)$ of 79 ESC cases.

The association of PD-L1 positivity by TPS and clinicopathological variables in ESC cases was analyzed. A statistical correlation was found between PD-L1 positivity by TPS and clinicopathological parameters, including age $\leq 63$ years $(p=0.025)$, stage II-IV $(p=0.015)$, myometrial invasion $\geq 50 \%(\mathrm{p}=0.001)$, and presence of LVSI $(p=0.002)$. However, there was no significant difference between PD-L1 positivity by CPS and clinicopathological parameters (Table 1).

\section{Prognostic Significance of PD-LI Expression and Clinicopathological}

\section{Features in ESCs}

Univariate analysis showed that PD-L1 positivity by TPS (HR: 2.132; $\mathrm{p}=0.043$ ), elevated serum CA125 (HR: 2.458; $\mathrm{p}=0.019$ ), advanced stage (HR: 5.276; $\mathrm{p}<0.001$ ), myometrial invasion $\geq 50 \%$ (HR: $2.925 ; p=0.004$ ), LVSI (HR: 2.319; $\mathrm{p}=0.032$ ), and positive ascites (HR: 2.661; $\mathrm{p}=$ 0.034 ) were related to poor OS in patients with ESC. Variables with $\mathrm{p}<0.05$ in the univariate analysis were used in the multivariable model by using an enter and forward stepwise selection. Only advanced stage (HR: $4.491 ; \mathrm{p}=0.001$ ) and myometrial invasion $\geq 50 \%$ (HR: 
Table 2 Univariate and Multivariate Analysis of the Clinicopathologic Parameters and OS

\begin{tabular}{|c|c|c|c|c|c|}
\hline \multirow[t]{2}{*}{ Variable } & \multicolumn{2}{|c|}{ Univariate } & \multicolumn{3}{|c|}{ Multivariate (Enter/[Forward] Selection) } \\
\hline & HR & p-value & HR & $\mathbf{C l}$ & p-value \\
\hline Age $(\leq 63$ vs $>63$ years $)$ & 1.038 & 0.919 & - & - & - \\
\hline BMI $\left(<25\right.$ vs $\left.\geq 25 \mathrm{~kg} / \mathrm{m}^{2}\right)$ & 0.877 & 0.731 & - & - & - \\
\hline CAI25 ( $\leq 35$ vs $>35 \mathrm{U} / \mathrm{mL})$ & 2.458 & $0.019^{\dagger}$ & 1.313 & $0.54 I-3.185$ & 0.547 \\
\hline Histotype (Pure vs Mixed) & 1.082 & 0.833 & - & - & - \\
\hline \multirow[t]{2}{*}{ Stage (I vs II-IV) } & 5.276 & $<0.00 \mathrm{I}^{\dagger}$ & 3.813 & $1.427-10.189$ & $0.008^{\dagger}$ \\
\hline & & & {$[4.49]$} & $1.795-11.235$ & $\left.0.001^{\dagger}\right]$ \\
\hline \multirow[t]{2}{*}{$\mathrm{Ml}(<50 \%$ vs $\geq 50 \%)$} & 2.925 & $0.004^{\dagger}$ & 1.971 & $0.846-4.593$ & 0.116 \\
\hline & & & {$[2.192$} & $1.040-4.616$ & $\left.0.039^{\dagger}\right]$ \\
\hline LNM (Absent vs Present) & 1.734 & 0.206 & - & - & - \\
\hline LVSI (Absent vs Present) & 2.319 & $0.032^{\dagger}$ & 1.073 & $0.423-2.718$ & 0.882 \\
\hline $\mathrm{RD}(\leq \mathrm{I}$ vs $>\mathrm{I} \mathrm{cm})$ & 2.432 & 0.099 & - & - & - \\
\hline Ascites (Absent vs Present) & 2.661 & $0.034^{\dagger}$ & 1.189 & $0.404-3.499$ & 0.754 \\
\hline TPS of PD-LI (Negative vs Positive) & 2.132 & $0.043^{\dagger}$ & 1.229 & $0.531-2.843$ & 0.631 \\
\hline CPS of PD-LI (Negative vs Positive) & 1.167 & 0.722 & - & - & - \\
\hline
\end{tabular}

Note: ${ }^{\dagger} \mathrm{p}<0.05$, Cox regression was used for multivariate analysis, with enter and forward selection.

Abbreviations: BMI, body mass index; MI, myometrial invasion; LNM, lymph node metastasis; LVSI, lymphovascular space invasion; RD, residual disease; TPS, tumor proportion score; $\mathrm{CPS}$, combined positive score; $\mathrm{HR}$, hazard ratio; $\mathrm{Cl}$, confidence interval.

$2.192 ; p=0.039)$ were found to be the prognostic factors of poor OS in the final multivariate Cox model (Table 2).

The univariate analysis showed that poor PFS was linked with PD-L1 positivity by TPS (HR: 2.380; $\mathrm{p}=$ 0.005), elevated serum CA125 (HR: 2.312; $\mathrm{p}=0.009$ ), advanced stage (HR: $3.139 ; \mathrm{p}<0.001$ ), myometrial invasion $\geq 50 \%$ (HR: 2.046; $\mathrm{p}=0.019$ ), lymph node metastasis (HR: 2.134, $\mathrm{p}=0.032$ ), LVSI (HR: 2.149; $\mathrm{p}=0.019$ ), residual disease $>1 \mathrm{~cm}(\mathrm{HR}: 2.888 ; \mathrm{p}=0.017)$, and positive ascites (HR: 3.361; p = 0.002). Meanwhile, parameters adjusted by the univariate model were enrolled for analyzing in multivariate Cox model, and the results showed that PD-L1 positivity by TPS (HR: 1.921; p = 0.039) and advanced stage (HR: 2.724; $\mathrm{p}=0.003$ ) were independent prognostic factors of poor PFS (Table 3 ).

\section{PD-LI Expression Inversely Correlates with PFS and OS in ESCs}

Available complete follow-up information was included in a survival analysis for 79 patients with ESC. The median follow-up time was 66 months (range from 6 to 168). Kaplan-Meier survival analysis showed that patients with PD-L1-positive tumors suffered significantly worse OS, when compared with PD-L1 negative tumors (log-rank $\mathrm{p}=0.037$ ) (Figure 2A). Similarly, patients with PD-L1 positive tumors suffered significantly worse PFS than did those with PD-L1-negative tumors ( $p=0.003$; Log rank test) (Figure 2C). In contrast, PD-L1 positivity by CPS within the ESC cases showed no statistical significance for OS and PFS (Figure 2B and D).

\section{Discussion}

Immunotherapy has been applied in clinical pathology at a robust rate due to promising effects in numerous solid tumors, including gynecologic tumors. ${ }^{23-25}$ However, the role of PD-L1 expression has not been clear in ESCs, largely due to limited available survival-based evidence. In this study, we analyzed PD-L1 expression in ESC tumors and correlated its expression with clinicopathologic parameters and survival outcomes to enhance our understanding of the role of the PD-1/PD-L1 pathway as a potential therapeutic target for this kind of tumor.

In our cohort, the prevalence of PD-L1 positivity in ESCs was $43.0 \%$ by TPS (cut-off $1 \%$ ) and $73.4 \%$ by CPS (cut-off 1). In addition, PD-L1 positivity by TPS was linked to a poorer outcome in ESC patients, and it was an independent prognostic factor. However, PD-L1 positivity by CPS within the ESC cases showed no statistical significance for OS and PFS. Currently, studies on the association between PD-L1 positivity and survival from ESC are lacking. We could not correlate our ESC-specific data to other studies, as none of the other groups separately analyzed this tumor type. Notably, Gulecet et al demonstrated that PD-L1 positivity (cut off $>5 \%$ tumor cells) was an independent poor predictor of non-endometrioid endometrial cancers. PD-L1 expression in tumor cells was 
Table 3 Univariate and Multivariate Analysis of the Clinicopathologic Parameters and PFS

\begin{tabular}{|c|c|c|c|c|c|}
\hline \multirow[t]{2}{*}{ Variable } & \multicolumn{2}{|c|}{ Univariate } & \multicolumn{3}{|c|}{ Multivariate (Enter/[Forward] Selection) } \\
\hline & HR & p-value & HR & $\mathbf{C l}$ & p-value \\
\hline Age ( $\leq 63$ vs $>63$ years $)$ & 0.724 & 0.297 & - & - & - \\
\hline BMI $\left(<25\right.$ vs $\left.\geq 25 \mathrm{~kg} / \mathrm{m}^{2}\right)$ & 0.859 & 0.630 & - & - & - \\
\hline CAI25 ( $\leq 35$ vs $>35 \mathrm{U} / \mathrm{mL})$ & 2.312 & $0.009^{\dagger}$ & 1.459 & $0.684-3.112$ & 0.328 \\
\hline Histotype (Pure vs Mixed) & 1.000 & 1.000 & - & - & - \\
\hline \multirow[t]{2}{*}{ Stage (I vs II-IV) } & 3.139 & $<0.001^{\dagger}$ & 2.637 & $1.206-5.767$ & $0.015^{\dagger}$ \\
\hline & & & {$[2.724$} & $|.4||-5.26|$ & $\left.0.003^{\dagger}\right]$ \\
\hline $\mathrm{Ml}(<50 \%$ vs $\geq 50 \%)$ & 2.046 & $0.019^{\dagger}$ & 1.757 & $0.824-3.747$ & 0.145 \\
\hline LNM (Absent vs Present) & 2.134 & $0.032^{\dagger}$ & 0.593 & $0.200-1.757$ & 0.346 \\
\hline LVSI (Absent vs Present) & 2.149 & $0.019^{\dagger}$ & 1.272 & $0.518-3.120$ & 0.599 \\
\hline $\mathrm{RD}(\leq \mathrm{I}$ vs $>\mathrm{I} \mathrm{cm})$ & 2.888 & $0.017^{\dagger}$ & 0.580 & $0.178-1.895$ & 0.367 \\
\hline Ascites (Absent vs Present) & 3.361 & $0.002^{\dagger}$ & 2.879 & $0.992-8.355$ & 0.052 \\
\hline \multirow[t]{2}{*}{ TPS of PD-LI (Negative vs Positive) } & 2.380 & $0.005^{\dagger}$ & 2.433 & $0.986-6.005$ & 0.054 \\
\hline & & & {$[1.921$} & $1.035-3.566$ & $\left.0.039^{\dagger}\right]$ \\
\hline CPS of PD-LI (Negative vs Positive) & 0.969 & 0.929 & - & - & - \\
\hline
\end{tabular}

Note: ${ }^{\dagger} \mathrm{p}<0.05$, Cox regression was used for multivariate analysis, with enter and forward selection model.

Abbreviations: BMI, body mass index; MI, myometrial invasion; LNM, lymph node metastasis; LVSI, lymphovascular space invasion; RD, residual disease; TPS, tumor proportion score; CPS, combined positive score; $\mathrm{HR}$, hazard ratio; $\mathrm{Cl}$, confidence interval.

associated with shorter OS, but PD-L1 expression in immune cells was not related with OS. ${ }^{14}$

In addition, we found that elevated serum CA125, advanced stage, deep myometrial invasion, lymph node metastasis, LVSI, residual disease $>1 \mathrm{~cm}$ and positive ascites were independent prognostic factors in ESC patients. Comparable findings have been reported elsewhere. Olawaiyeet et al reported that elevated serum
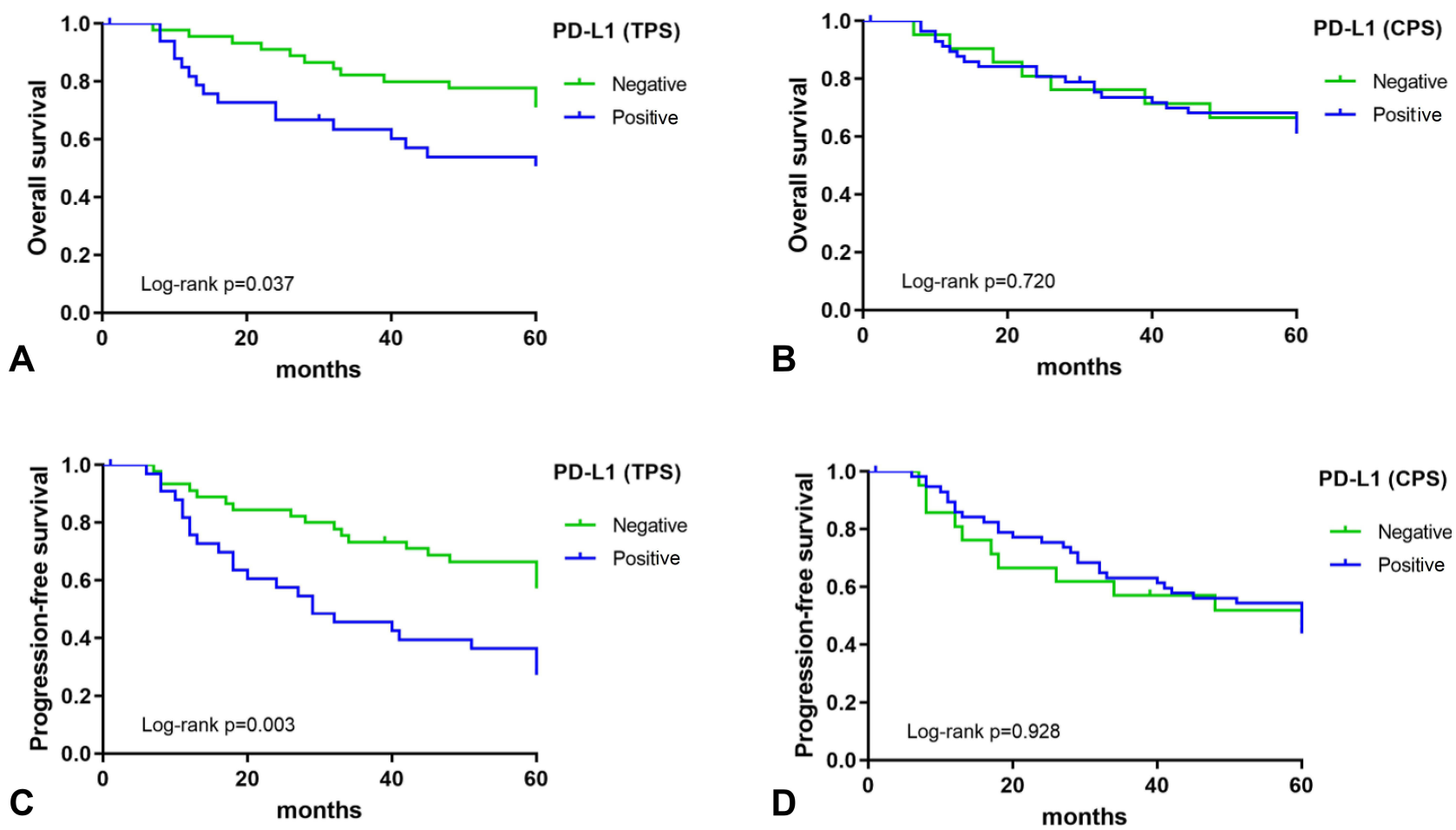

Figure 2 Kaplan-Meier curves of overall survival (OS) and progression-free survival (PFS) in patients with endometrial serous carcinoma (ESC). (A) OS in PD-LI-negative group (green) and PD-LI-positive group (blue) using tumor proportion score (TPS) (cut off I\%). (B) OS in PD-LI-negative group (green) and PD-LI-positive group (blue) using combined positive score (CPS) (cut off I). (C) PFS in PD-LI-negative group (green) and PD-LI-positive group (blue) using TPS (cut off I\%). (D) PFS in patients in PD-LI -negative group (green) and PD-LI-positive group (blue) using CPS (cut off I). 
CA125 levels correlated with advanced stage of ESC. ${ }^{26}$ Some studies have observed that deep myometrial invasion was linked with worse outcome in ESC patients. ${ }^{27-29}$ The incidence of lymph node metastasis is more common in ESC than in endometrioid carcinoma, ${ }^{30-33}$ and lymphadenectomy was associated with improved OS in ESC patients. ${ }^{34}$ Studies have demonstrated that LVSI was an independent prognostic factor for ESC patients. ${ }^{35,36} \mathrm{Garg}$ et al showed that the risk of death was 4.3 times higher among stage IA ESC patients with positive peritoneal cytology compared to the negative cytology group. ${ }^{37}$ Our findings are similar to previously published observations that optimal cytoreduction correlates with improved outcomes for patients with ESC. ${ }^{38-40}$ Of note, we found that PD-L1 positivity in tumor cells was significantly correlated with advanced stage, deep myometrial invasion and LVSI.

Our study has several strengths relative to the previous studies. To our knowledge, our study is the first of this kind to focus on a large cohort of ESCs, especially considering the relative rarity of this kind of tumor. We utilized whole tissue sections to reduce sampling bias because of the frequent PD-L1 intra-tumor heterogeneity. Moreover, the genomic data from The Cancer Genome Atlas (TCGA) defined endometrial cancer types into four molecular subgroups: polymerase epsilon (POLE) ultramutated, microsatellite instability, copy-number low, and copy-number high. $^{41}$ Most of the copy-number-high tumors had TP53 mutations and diagnosed as high-grade serous cancers. Currently, analysis of TP53 mutations or p53 expression by IHC has been suggested as a reliable marker for these tumors. ${ }^{42}$ Of note, all ESC cases studied here were p53 mutant type by IHC analysis. Thus, our data may potentially provide clinically useful information benefitting those copy-number-high tumors with PD-L1 expression.

Our study also has limitations, notably that 34 mixed ESC cases were included and the lack of external validation in this ESC cohort. Such lack of external validation reflects the novelty of this study. Potential biases were also present, which were caused by other residual confounding factors, such as not adjusting ESC cases by TCGA classification, diabetes status, etc. Therefore, ESC areas should be confirmed by analysis of TP53 mutations in addition to p53 expression by IHC in the future. Intra-tumoral lymphocytes and macrophages usually have membranous staining, which may be misinterpreted as tumor cells. Future studies with a larger cohort and new testing such as multiplex IHC will be needed to further validate our findings.

\section{Conclusion}

In summary, PD-L1 is commonly expressed in tumor cells as well as in immune cells within the ESC cases we studied. Such observation suggests that immune therapy by targeting the PD-1/PD-L1 pathway may represent an alternative therapeutic choice for ESC. This may be particularly useful for those cases showing PD-L1 expression in ESC tumor cells because patients with such tumors have a worse outcome than those patients with negative PD-L1 expression.

\section{Abbreviations}

ESC, endometrial serous carcinoma; PD-L1, programmed death-ligand 1; IHC, immunohistochemistry; BMI, body mass index; MI, myometrial invasion; LNM, lymph node metastasis; LVSI, lymphovascular space invasion; $\mathrm{RD}$, residual disease; TPS, tumor proportion score; CPS, combined positive score; OS, overall survival; PFS, progression-free survival; HR, hazard ratio; CI, confidence interval.

\section{Data Sharing Statement}

The datasets generated or analyzed during the current study available from the corresponding author on reasonable request.

\section{Ethics Approval and Consent to Participate}

The study was approved by the Institutional Research Ethics Board at Zhejiang University School of Medicine Women's Hospital, China (No: IRB-20200281-R). All procedures performed in studies involving human participants were in accordance with the ethical standards of the institutional and/or national research committee and with the Helsinki declaration. Each enrolled patient permitted and signed up informed consent to use their samples and records for scientific research.

\section{Acknowledgments}

We are grateful to Junjie Zhu (Department of Epidemiology, School of Public Health, Fudan University, Shanghai, China/Department of Epidemiology and Health Statistics, School of Public Health, Dali University, Dali, China) for statistical technical assistance. 


\section{Author Contributions}

All authors made a significant contribution to the work reported, whether that is in the conception, study design, execution, acquisition of data, analysis and interpretation, or in all these areas; took part in drafting, revising or critically reviewing the article; gave final approval of the version to be published; have agreed on the journal to which the article has been submitted; and agree to be accountable for all aspects of the work.

\section{Funding}

This work was supported by the Scientific Research Fund of Zhejiang Provincial Education Department (No. Y202045491) and the Medicine and Health of Zhejiang Provincial Technology Plan Project (No. 2021KY766).

\section{Disclosure}

The authors state they have no competing interests.

\section{References}

1. Siegel RL, Miller KD, Jemal A. Cancer statistics. CA Cancer J Clin. 2020;70(1):7-30. doi:10.3322/caac.21590

2. Lu KH, Broaddus RR, Longo DL. Endometrial cancer. $N$ Engl J Med. 2020;383(21):2053-2064. doi:10.1056/NEJMra1514010

3. Del Carmen MG, Birrer M, Schorge JO. Uterine papillary serous cancer: a review of the literature. Gynecol Oncol. 2012;127 (3):651-661. doi:10.1016/j.ygyno.2012.09.012

4. World Health Organization. Classification of Tumours: Female Genital Tumours. 5th ed. Lyon (France): International Agency for Research on Cancer; 2020.

5. Zhang L, Kwan SY, Wong KK, Solaman PT, Lu KH, Mok SC. Pathogenesis and clinical management of uterine serous carcinoma. Cancers. 2020;12(3):686. doi:10.3390/cancers 12030686

6. Schwab CL, Santin AD. Targeted therapy in the treatment of uterine serous carcinoma. Pharmacogenomics. 2015;16(2):97-99. doi:10.2 217/pgs. 14.176

7. Heim D, Budczies J, Stenzinger A, et al. Cancer beyond organ and tissue specificity: next-generation-sequencing gene mutation data reveal complex genetic similarities across major cancers. Int J Cancer. 2014;135(10):2362-2369. doi:10.1002/ijc.28882

8. Hoadley KA, Yau C, Wolf DM, et al. Multiplatform analysis of 12 cancer types reveals molecular classification within and across tissues of origin. Cell. 2014;158(4):929-944. doi:10.1016/j.cell.2014.06.049

9. Zhong H, Chen H, Qiu H, Huang C, Wu Z. A multiomics comparison between endometrial cancer and serous ovarian cancer. PeerJ. 2020;8e8347. doi: $10.7717 /$ peerj. 8347

10. Matulonis UA, Shapira-Frommer R, Santin AD, et al. Antitumor activity and safety of pembrolizumab in patients with advanced recurrent ovarian cancer: results from the Phase II KEYNOTE-100 study. Ann Oncol. 2019;30(7):1080-1087. doi:10.1093/annonc/ mdz135

11. Sharpe AH, Pauken KE. The diverse functions of the PD1 inhibitory pathway. Nat Rev Immunol. 2018;18(3):153-167. doi:10.1038/ nri.2017.108

12. Sun C, Mezzadra R, Schumacher TN. Regulation and Function of the PD-L1 Checkpoint. Immunity. 2018;48(3):434-452. doi:10.1016/j. immuni.2018.03.014
13. Sungu N, Yildirim M, Desdicioglu R, et al. Expression of immunomodulatory molecules PD-1, PD-L1, and PD-L2, and their relationship with clinicopathologic characteristics in endometrial cancer. Int J Gynecol Pathol. 2019;38(5):404-413. doi:10.1097/PGP.00 00000000000543

14. Kucukgoz Gulec U, Kilic Bagir E, Paydas S, Guzel AB, Gumurdulu D, Vardar MA. Programmed death-1 (PD-1) and programmed death-ligand 1 (PD-L1) expressions in type 2 endometrial cancer. Arch Gynecol Obstet. 2019;300(2):377-382. doi:10.1007/ s00404-019-05180-2

15. Kim J, Kim S, Lee HS, et al. Prognostic implication of programmed cell death 1 protein and its ligand expressions in endometrial cancer. Gynecol Oncol. 2018;149(2):381-387. doi:10.1016/j.ygyno.2018.02.013

16. Pasanen A, Ahvenainen T, Pellinen T, Vahteristo P, Loukovaara M, Butzow R. PD-L1 expression in endometrial carcinoma cells and intratumoral immune cells: differences across histologic and TCGA-based molecular subgroups. Am J Surg Pathol. 2020;44 (2):174-181. doi:10.1097/PAS.0000000000001395

17. Sloan EA, Ring KL, Willis BC, Modesitt SC, Mills AM. PD-L1 expression in mismatch repair-deficient endometrial carcinomas, including lynch syndrome-associated and MLH1 promoter hypermethylated tumors. Am J Surg Pathol. 2017;41(3):326-333. doi:10.1097/PAS.0000000000000783

18. Li Z, Joehlin-Price AS, Rhoades J, et al. Programmed death ligand 1 expression among 700 consecutive endometrial cancers: strong association with mismatch repair protein deficiency. Int $J$ Gynecol Cancer. 2018;28(1):59-68. doi:10.1097/IGC.0000000000001120

19. Talhouk A, Derocher H, Schmidt P, et al. Molecular subtype not immune response drives outcomes in endometrial carcinoma. Clin Cancer Res. 2019;25(8):2537-2548. doi:10.1158/1078-0432.CCR18-3241

20. Makker V, Rasco D, Vogelzang NJ, et al. Lenvatinib plus pembrolizumab in patients with advanced endometrial cancer: an interim analysis of a multicentre, open-label, single-arm, Phase 2 trial. Lancet Oncol. 2019;20(5):711-718. doi:10.1016/s1470-2045(19) 30020-8

21. Chen H, Molberg K, Strickland AL, et al. PD-L1 expression and CD8 + tumor-infiltrating lymphocytes in different types of tubo-ovarian carcinoma and their prognostic value in high-grade serous carcinoma. Am J Surg Pathol. 2020;44(8):1050-1060. doi:10.1097/PAS.00 00000000001503

22. Davis AA, Patel VG. The role of PD-L1 expression as a predictive biomarker: an analysis of all US Food and Drug Administration (FDA) approvals of immune checkpoint inhibitors. $J$ Immunother Cancer. 2019;7(1):278. doi:10.1186/s40425-019-0768-9

23. Pham T, Roth S, Kong J, et al. An update on immunotherapy for solid tumors: a review. Ann Surg Oncol. 2018;25(11):3404-3412. doi:10.1245/s10434-018-6658-4

24. Liao JB. Immunotherapy for gynecologic cancers. Gynecol Oncol. 2016;142(1):3-5. doi:10.1016/j.ygyno.2016.05.029

25. Schepisi G, Casadei C, Toma I, et al. Immunotherapy and its development for gynecological (ovarian, endometrial and cervical) tumors: from immune checkpoint inhibitors to chimeric antigen receptor (CAR)-T cell therapy. Cancers. 2021;13(4):840. doi:10.3390/ cancers 13040840

26. Olawaiye AB, Rauh-Hain JA, Withiam-Leitch M, Rueda B, Goodman A, Del Carmen MG. Utility of pre-operative serum CA-125 in the management of uterine papillary serous carcinoma. Gynecol Oncol. 2008;110 (3):293-298. doi:10.1016/j.ygyno.2008.05.027

27. Slomovitz BM, Burke TW, Eifel PJ, et al. Uterine papillary serous carcinoma (UPSC): a single institution review of 129 cases. Gynecol Oncol. 2003;91(3):463-469. doi:10.1016/j.ygyno.2003.08.018

28. Van Der Putten LJ, Hoskins P, Tinker A, Lim P, Aquino-Parsons C, Kwon JS. Population-based treatment and outcomes of Stage I uterine serous carcinoma. Gynecol Oncol. 2014;132(1):61-64. doi:10.1016/j.ygyno.2013.11.002 
29. Solmaz U, Mat E, Ekin A, et al. Optimal cytoreduction, depth of myometrial invasion, and age are independent prognostic factors for survival in women with uterine papillary serous and clear cell carcinomas. Int $J$ Surg. 2016:3271-3377. doi:10.1016/j. ijsu.2016.06.041

30. Mattes MD, Lee JC, Metzger DJ, Ashamalla H, Katsoulakis E. The incidence of pelvic and para-aortic lymph node metastasis in uterine papillary serous and clear cell carcinoma according to the SEER registry. J Gynecol Oncol. 2015;26(1):19-24. doi:10.3802/ jgo.2015.26.1.19

31. Wang Y, Yu M, Yang JX, Cao DY, Shen K, Lang JH. Clinicopathological and survival analysis of uterine papillary serous carcinoma: a single institutional review of 106 cases. Cancer Manag Res. 2018;104915-104928. doi:10.2147/CMAR.S179566

32. Karalok A, Turan T, Basaran D, et al. Lymph node metastasis in patients with endometrioid endometrial cancer: overtreatment is the main issue. Int J Gynecol Cancer. 2017;27(4):748-753. doi:10.1097/ IGC.0000000000000937

33. Lee J, Kong TW, Paek J, Chang SJ, Ryu HS. Predicting model of lymph node metastasis using preoperative tumor grade, transvaginal ultrasound, and serum CA-125 level in patients with endometrial cancer. Int J Gynecol Cancer. 2016;26(9):1630-1635. doi:10.1097/ IGC.0000000000000820

34. Mahdi H, Elshaikh MA, Debenardo R, et al. Impact of adjuvant chemotherapy and pelvic radiation on pattern of recurrence and outcome in stage I non-invasive uterine papillary serous carcinoma. A multi-institution study. Gynecol Oncol. 2015;137(2):239-244. doi:10.1016/j.ygyno.2015.01.544
35. Winer I, Ahmed QF, Mert I, et al. Significance of lymphovascular space invasion in uterine serous carcinoma. Int J Gynecol Pathol. 2015;34(1):47-56. doi:10.1097/pgp.0000000000000113

36. Solmaz U, Ekin A, Mat E, et al. Analysis of clinical and pathological characteristics, treatment methods, survival, and prognosis of uterine papillary serous carcinoma. Tumori. 2016;102(6):593-599. doi: $10.5301 / \mathrm{tj} .5000531$

37. Garg G, Gao F, Wright JD, Hagemann AR, Mutch DG, Powell MA. Positive peritoneal cytology is an independent risk-factor in early stage endometrial cancer. Gynecol Oncol. 2013;128(1):77-82. doi:10.1016/j.ygyno.2012.09.026

38. Bristow RE, Duska LR, Montz FJ. The role of cytoreductive surgery in the management of stage IV uterine papillary serous carcinoma. Gynecol Oncol. 2001;81(1):92-99. doi:10.1006/gyno.2000.6110

39. Patsavas K, Woessner J, Gielda B, et al. Optimal surgical debulking in uterine papillary serous carcinoma affects survival. Gynecol Oncol. 2011;121(3):581-585. doi:10.1016/j.ygyno.2010.11.048

40. Thomas MB, Mariani A, Cliby WA, Keeney GL, Podratz KC, Dowdy SC. Role of cytoreduction in stage III and IV uterine papillary serous carcinoma. Gynecol Oncol. 2007;107(2):190-193. doi:10.1016/j.ygyno.2007.05.039

41. Kandoth C, Schultz N, Cherniack AD, et al.; Cancer Genome Atlas Research N. Integrated genomic characterization of endometrial carcinoma. Nature. 2013;497(7447):67-73. doi:10.1038/nature12113

42. Talhouk A, Mcconechy MK, Leung S, et al. A clinically applicable molecular-based classification for endometrial cancers. Br J Cancer. 2015;113(2):299-310. doi:10.1038/bjc.2015.190

\section{Publish your work in this journal}

Cancer Management and Research is an international, peer-reviewed open access journal focusing on cancer research and the optimal use of preventative and integrated treatment interventions to achieve improved outcomes, enhanced survival and quality of life for the cancer patient.
The manuscript management system is completely online and includes a very quick and fair peer-review system, which is all easy to use. Visit http://www.dovepress.com/testimonials.php to read real quotes from published authors. 